\section{Indicadores de prescrição médica \\ nas unidades básicas de Saúde da \\ Família no município de Campina \\ Grande, PB}

\section{Prescription indicators in Family Health basic units of the municipal district of Campina Grande, PB}

Andrezza Duarte Farias'

Maria Aparecida Alves Cardoso ${ }^{2,3}$

Ana Cláudia Dantas de Medeiros ${ }^{3}$

Lindomar de Farias Belém ${ }^{3}$

Mônica de Oliveira da Silva Simões ${ }^{3}$

${ }^{1}$ Secretaria de Saúde do Município de Campina Grande

${ }^{2}$ Núcleo de Estudos e Pesquisas Epidemiológicas - NEPE/Universidade Estadual da Paraíba (UEPB)

${ }^{3}$ Departamento de Farmácia, Universidade Estadual da Paraíba (UEPB)

Correspondência: Andrezza Duarte Farias. Travessa Vigário Calixto, 889 Bairro Catolé - Campina Grande, PB - CEP 58104-480. E-mail: andrezza_duarte@hotmail.com

\section{Resumo}

Introdução: O Sistema Único de Saúde (SUS) assegura o acesso aos medicamentos, mediante a garantia da execução integral da Assistência Farmacêutica. A Organização Mundial de Saúde (OMS) desenvolveu os Indicadores do Uso de Medicamentos com o intuito de descrever e avaliar aspectos que afetam a prática farmacêutica nos centros de saúde. Objetivo: Conhecer $\mathrm{a}(\mathrm{s})$ classes terapêuticas mais prescritas, segundo os indicadores de prescrição médica nas Unidades de Saúde da Família (UBSF) do município de Campina Grande. Métodos: O estudo baseou-se nos Indicadores de Prescrição de Medicamentos propostos pela OMS. Os medicamentos foram classificados segundo a Anatomical Therapeutic Chemical Classification (ATC) e os dados analisados utilizando-se os programas EpiInfo 2000 e Excel. Resultados: Foi prescrita uma média de 1,5 medicamentos por receita médica. A porcentagem de antibióticos prescritos foi de $21,1 \%$. Os medicamentos foram prescritos pelo nome genérico em $84,2 \%$ das prescrições e apenas $1,1 \%$ eram injetáveis. Faziam parte da lista de medicamentos padronizados $91,9 \%$ dos prescritos. O grupo farmacológico mais prescrito foi o de antibióticos $(21,0 \%)$, seguido dos antiparasitários $(18,4 \%)$, analgésicos e antipiréticos $(15,4 \%)$, medicamentos para o aparelho digestivo $(9,5 \%)$ e respiratório $(9,2 \%)$. Conclusões: Percebe-se a importância de conhecer as principais demandas da comunidade, a fim de que os serviços possam planejar e realizar intervenções pertinentes às necessidades da população. Os indicadores apresentaram bons índices, demonstrando possível conseqüência da Política Nacional de Medicamentos e da realização da Conferência Municipal de Medicamentos e Assistência Farmacêutica.

Palavras-chave: Medicamentos. Assistência Farmacêutica. Indicadores de Prescrição. Atenção Básica. 
Abstract

Introduction: The "Sistema Único de Saúde" (Unified National Health System SUS) ensures access to medication through the guarantee of integral pharmaceutical care. The World Health Organization (WHO) developed Indicators on the Utilization of Drugs with the intention of describing and evaluating aspects that affect pharmaceutical practices in health centers. Objectives: To learn which are the most commonly prescribed therapeutic groups, according to Prescription Indicators in the Family Health Program of the municipal district of Campina Grande. Methods: The study was based on the Prescription Indicators proposed by the WHO. Drugs were classified according to the Anatomical Therapeutic Chemical Classification (ATC) and data were analyzed using EpiInfo 2000 and Excel programs. Results: An average of 1.5 drugs were prescribed per prescription. The percentage of antibiotics prescribed was $21.1 \%$. Medication was prescribed by its generic name in $84.2 \%$ of prescriptions and only $1.1 \%$ was parenteral. $91.9 \%$ of the medications prescribed were part of the list of standard drugs. The most frequently prescribed pharmacologic group was antibiotics (21.0\%), followed by anti-parasites (18.4\%), analgesics and antithermals (15.4\%), and drugs for the digestive $(9.5 \%)$ and respiratory (9.2\%) systems. Conclusions: It is important to know the community's main demands, so that policy-makers can plan and carry out appropriate activities to meet the needs of the population. Indicators presented good indexes, possibly as a consequence of the National Drug Policy and the accomplishment of the Municipal Conference on Medication and Pharmaceutical Care.

Keywords: Medicines. Pharmaceutical Care. Prescription Indicators. Basic Care. Health Services.

\section{Introdução}

O Sistema Único de Saúde (SUS) assegura $\mathrm{o}$ acesso aos medicamentos, mediante a garantia da execução integral da Assistência Farmacêutica ${ }^{1}$. Esta é definida como "o grupo de atividades relacionadas com o medicamento, destinadas a apoiar as ações de saúde demandadas por uma comunidade. Envolve o abastecimento de medicamentos em todas e em cada uma de suas etapas constitutivas, a conservação e o controle de qualidade, a segurança e a eficácia terapêutica dos medicamentos, o acompanhamento e a avaliação da utilização, a obtenção e a difusão de informação sobre medicamentos e a educação permanente dos profissionais de saúde, do paciente e da comunidade para assegurar o uso racional de medicamentos." ${ }^{2}$

Buscando realizar o promulgado na Lei Orgânica da Saúde (LOS), durante os últimos anos o setor farmacêutico tem passado por diversas transformações, destacando-se a Política Nacional de Medicamentos (PNM), a criação da Agência Nacional de Vigilância Sanitária (ANVISA), a Conferência Nacional de Medicamentos e Assistência Farmacêutica (CNMAF) e a Política Nacional de Assistência Farmacêutica $(\mathrm{PNAF})^{1-3}$.

A Política Nacional de Medicamentos (PNM) constitui o principal instrumento para a orientação das ações de saúde relacionadas aos mesmos. Apresenta como principais objetivos "garantir a necessária segurança, eficácia e qualidade dos medicamentos, a promoção do uso racional e o acesso da população àqueles considerados essenciais" ${ }^{2}$.

Para tanto, possui algumas prioridades, entre as quais está a promoção do Uso Racional de Medicamentos, que "compreende a prescrição apropriada; a disponibilidade oportuna e a preços acessíveis; a dispensação em condições adequadas; e o consumo nas doses indicadas, nos intervalos definidos e no período de tempo indicado de medicamentos eficazes, seguros e de qualidade" ${ }^{2}$. 
A prescrição ou receita médica é um instrumento essencial para a terapêutica e para o Uso Racional de Medicamentos, pois deve conter as informações necessárias sobre o medicamento: a dose, a freqüência e a duração do tratamento adequados para o(s) problema(s) do paciente. Trata-se, portanto, de um importante fator para a qualidade e quantidade do consumo de medicamentos, embora o ato da prescrição sofra influências do conhecimento do prescritor, das expectativas do paciente e da indústria farmacêutica ${ }^{2,4}$.

Diante da crescente preocupação em promover o Uso Racional de Medicamentos, a Organização Mundial de Saúde (OMS) desenvolveu os Indicadores do Uso de Medicamentos como uma maneira de descrever e avaliar com segurança aspectos que afetam a prática farmacêutica em grandes e pequenos centros de saúde ${ }^{5}$.

Os Indicadores de Prescrição permitem conhecer as práticas terapêuticas correntes, comparar parâmetros entre instituições similares e descrever as necessidades de medicamentos da população atendida ${ }^{6}$.

Os principais indicadores do uso de medicamentos são:

- Média de medicamentos por prescrição médica - objetiva medir o grau de polimedicação do paciente, visto ser este um dos fatores de interações medicamentosas e reações adversas. Permite observar a educação e a informação do prescritor ${ }^{5}$;

- Porcentagem de medicamentos prescritos pelo nome genérico - a prescrição de medicamentos pelo nome genérico propicia o controle dos custos de medicamentos no serviço de saúde, devido ao fato de os medicamentos de referência geralmente serem mais one$\operatorname{rosos}^{5,7}$;

- Porcentagem de medicamentos prescritos da lista de medicamentos essenciais - além do fator de controle dos custos, a lista de medicamentos essenciais tem por objetivo garantir o tratamento para as principais patologias da população. Dessa forma, este indicador possibilita verificar o grau de adequação das prescrições à padronização de medicamentos ${ }^{5}$;

- Porcentagem de antibióticos prescritos - o principal problema relacionado à prescrição excessiva e inapropriada de antibióticos é o desenvolvimento de resistência bacteriana, que pode acarretar graves conseqüências ao paciente ${ }^{5}$;

- Porcentagem de injetáveis prescritos apesar de importantes em diversas situações, a administração de injetáveis pode trazer sérias conseqüências quando erroneamente prescritos ou aplicados, como, por exemplo, dificuldade de reversão no caso de reações anafiláticas, reações adversas, necroses teciduais, etc. ${ }^{5}$

Em Campina Grande, a Secretaria Municipal de Saúde não possui uma divisão/ gerência destinada ao gerenciamento da Assistência Farmacêutica. A Farmácia Central (FACEN) é o setor responsável pelo armazenamento e distribuição de medicamentos e insumos farmacêuticos para os serviços de saúde do município: maternidade (Instituto de Saúde Elpídio de Almeida - ISEA), Serviço de Assistência Especializada (SAE), Centros de Apoio Psicossocial (CAPS), Serviço Ambulatorial de Urgência (SAMU), Centros e Unidades Básicas de Saúde e as Unidades Básicas de Saúde da Família (UBSFs).

Segundo a literatura, a organização da atenção básica é uma tendência mundial. No Brasil, o Programa Saúde na Família (PSF) e o Programa de Agentes Comunitários de Saúde (PACS), têm sido valorizados enquanto eixos centrais para a organização da atenção básica nos municípios ${ }^{8}$. O Programa Saúde da Família (PSF), implantado pelo Ministério da Saúde em 1994, visa o princípio da vigilância à saúde, que prioriza as ações de promoção, proteção e recuperação da saúde familiar, de forma integral e contínua. Possibilita, portanto, uma compreensão ampliada do processo saúde/doença e da necessidade de intervenções, além das práticas curativas ${ }^{9}$. 
O estudo teve por objetivo conhecer os medicamentos e as classe(s) terapêutica(s) mais prescritos, segundo os indicadores de prescrição médica nas Unidades Básicas de Saúde da Família do município de Campina Grande, pois não havia registro de informações sobre o uso de medicamentos no município. Nesse contexto, esses dados foram considerados de fundamental importância para a avaliação da atenção básica e posterior tomada de decisões pertinentes.

\section{Métodos}

A pesquisa, do tipo transversal, foi desenvolvida durante os meses de novembro e dezembro de 2005, utilizando como base os Indicadores de Prescrição de Medicamentos propostos pela Organização Mundial de Saúde (OMS) ${ }^{5}$.

Foram estudadas as Unidades Básicas de Saúde da Família (UBSF) da zona urbana do município de Campina Grande - PB. Para determinação da amostra foi seguida a orientação da Rapid Pharmaceutical Management Assessment (RPMA), que preconiza que sejam estudadas 30 prescrições médicas de 20 unidades de saúde ${ }^{10}$. Porém, diante de problemas de infra-estrutura e da desistência de participar de alguns profissionais de saúde, o estudo foi realizado em 11 Unidades Básicas de Saúde da Família (UBSF).

Sendo assim, a fonte de dados foi a receita médica, tendo procedido o sorteio entre as 31 UBSF do município. Foi solicitado aos médicos das unidades que prescrevessem as receitas em duas vias, sendo uma recolhida para a pesquisa e a outra para o paciente.

Os dados foram analisados através dos programas Epi Info 2000 e Excel. Os resultados foram apresentados de maneira descritiva e utilizando-se tabela e figuras, com intervalo de confiança de 95\% $(\mathrm{p}<0,05)$.

A pesquisa foi submetida à aprovação do Comitê de Ética e Pesquisa (CEP) da Universidade Estadual da Paraíba (UEPB) e recebeu parecer favorável. A Secretaria de Saúde de Campina Grande autorizou a realização da pesquisa e os dados foram expostos de forma agregada e sem a identificação das unidades de saúde pesquisadas, que foram codificadas por letras (A, B, C, D, E, F, G, H, I, J, L).

Foi utilizada a Relação Municipal de Medicamentos Essenciais (REMUME) vigente em 2005, para a identificação dos medicamentos padronizados e não padronizados, observando-se o princípio ativo, a concentração e forma farmacêutica.

Os medicamentos foram apresentados segundo a Denominação Comum Brasileira - DCB e classificados pelo Índice de Classificação Anatômico Químico e Terapêutico (ATC), para o conhecimento dos medicamentos e classes terapêuticas mais prescritas $^{11,12}$.

Os indicadores de prescrição recomendados pela OMS compõem as variáveis do estudo, que foram:

- o número de medicamentos por receita;

- porcentagem de medicamentos prescritos pelo nome genérico;

- porcentagem de medicamentos prescritos pertencentes à lista de medicamentos padronizados;

- porcentagem de antibióticos e

- porcentagem de injetáveis prescritos ${ }^{5}$.

\section{Resultados}

Ao final da pesquisa, foram coletadas 1.404 receitas, totalizando 2.166 medicamentos prescritos, o que contabiliza uma média de 1,5 medicamentos/ receita (Figura 1).

A porcentagem de antibióticos prescritos foi de $21,1 \%$. Os medicamentos foram prescritos pelo nome genérico em $84,2 \%$ das prescrições, e apenas $1,1 \%$ dos medicamentos eram injetáveis. Faziam parte da lista de medicamentos padronizados 91,9\% dos prescritos (Tabela 1).

O grupo farmacológico de maior representatividade foram os antibióticos $(21,0 \%)$, seguido dos antiparasitários $(18,4 \%)$, analgésicos e antipiréticos (15,4\%), medicamen- 


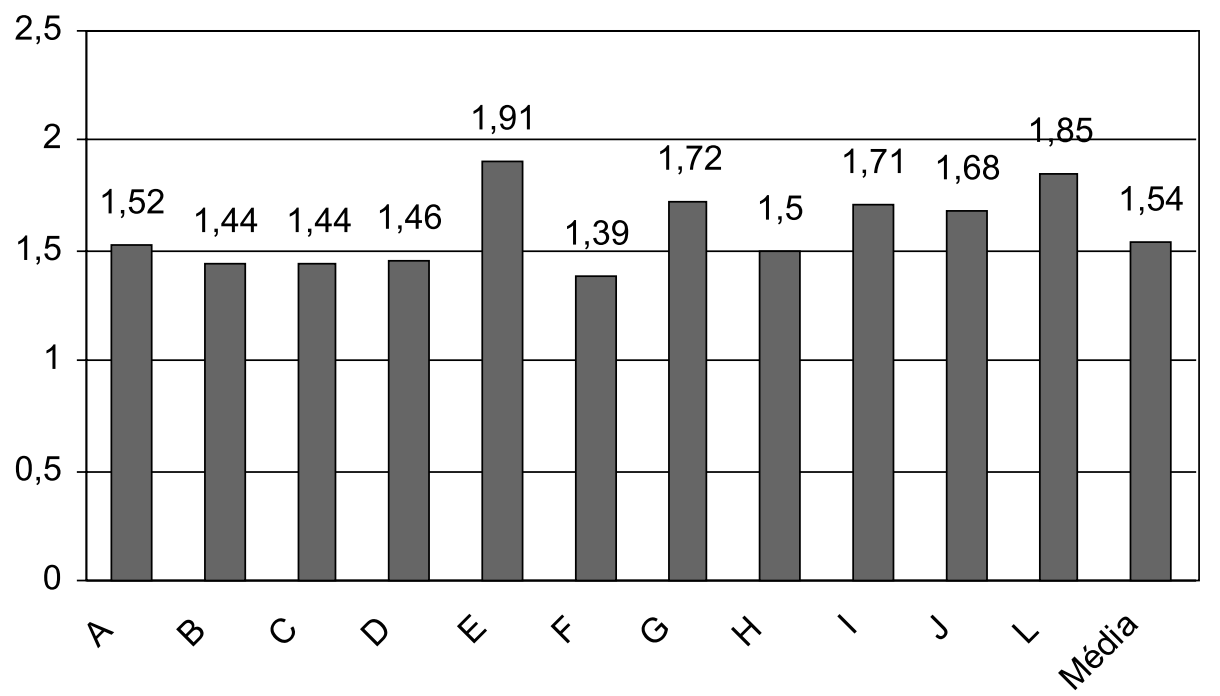

Figura 1 - Média de medicamentos prescritos por receita médica nas Unidades Básicas de Saúde da Família (UBSF) pesquisadas

Figure 1 - Average of medicines with medical prescription in the Basic Family Health Units (UBSF) surveyed.

Tabela 1 - Indicadores de Prescrição de Medicamentos segundo outros estudos desenvolvidos no país.

Table 1 - Medication Prescription Indicators according to other studies developed in the country.

\begin{tabular}{|c|c|c|c|c|c|c|c|c|}
\hline & $\begin{array}{l}\text { Campina Grande, } \\
\text { PB (2005) }\end{array}$ & Brasil (2005) & $\begin{array}{l}\text { Colombo et al } \\
\qquad \begin{array}{l}(2004) \\
\text { Blumenau, SC }\end{array}\end{array}$ & $\begin{array}{l}\text { Marcondes } \\
\text { (2002) } \\
\text { Ponta Grossa, PR }\end{array}$ & $\begin{array}{c}\text { Naves \& Silver } \\
\quad(2001) \\
\text { Brasília, DF }\end{array}$ & $\begin{array}{l}\text { Santos \& Nitirni } \\
\text { (1999) } \\
\text { Ribeirão Preto, SP }\end{array}$ & $\begin{array}{l}\text { Carneiro et al } \\
\text { (1999) } \\
\text { Américo } \\
\text { Brasiliense, SP }\end{array}$ & $\begin{array}{l}\text { OMS } \\
\text { recomendado }\end{array}$ \\
\hline $\begin{array}{l}\text { Média de } \\
\text { medicamentos } \\
\text { por receita médica }\end{array}$ & ca & 2,3 & 1,8 & 2,6 & 2,3 & 2,2 & 2,9 & $\begin{array}{l}2,0 \text { ou } \\
\text { menos }\end{array}$ \\
\hline $\begin{array}{l}\%^{1} \text { nome } \\
\text { genérico }\end{array}$ & 84,2 & 84,2 & $\begin{array}{c}\text { Não } \\
\text { coletado }\end{array}$ & 71 & 73,2 & 30,6 & 33,9 & 100 \\
\hline$\%$ LMP $^{2}$ & 91,9 & 78,3 & 82,4 & 87 & 85,3 & 83,4 & 54,1 & 70 \\
\hline$\%$ antibióticos & 21,1 & 40,1 & 12,5 & 33 & 26,4 & 21,3 & 31,3 & $\begin{array}{l}20 \text { ou } \\
\text { menos }\end{array}$ \\
\hline \% injetáveis & 1,1 & 7,9 & 8,1 & 13 & 7,5 & 8,3 & 9,9 & 10 \\
\hline
\end{tabular}

tos para o aparelho digestivo $(9,5 \%)$ e respiratório $(9,2 \%)$ (Figura 2).

\section{Discussão}

Relacionamos os valores obtidos com o de outros estudos, de forma a evidenciar a comparabilidade que a metodologia oferece e a importância do desenvolvimento de pesquisas semelhantes (Tabela 1).

$\mathrm{O}$ valor médio de medicamentos prescritos por receita neste estudo foi menor que o relatado por Colombo et al., que foi $1,8^{7}$; Santos \& Nitrini, $2,2^{13}$, Naves \& Silver, 


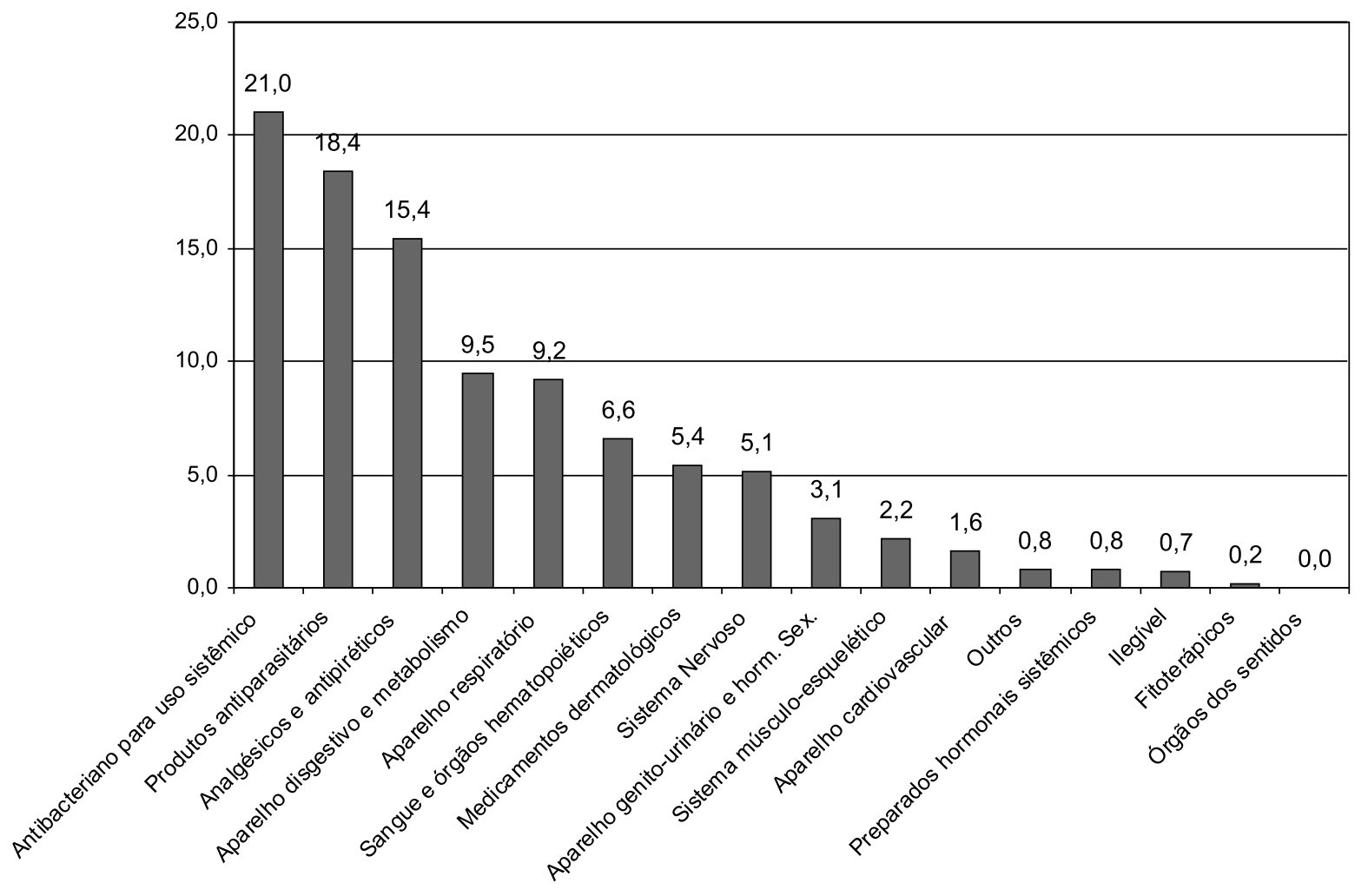

Figura 2 - Grupos farmacológicos mais prescritos nas UBSF pesquisadas

Figure 2 - Most frequently prescribed pharmacological groups in the UBSF surveyed

2,3 $3^{14}$, Marcondes, 2,6 $6^{15}$ e Carneiro et al, $2,9^{16}$, sendo que a média nacional encontrada foi de $2,3^{3}$. Isso demonstrou a racionalidade dos prescritores em relação à prescrição excessiva de medicamentos, protegendo a população de possíveis interações e reações adversas que podem ocorrer quando do uso concomitante de vários medicamentos ${ }^{10}$.

A prescrição pelo nome genérico ocorreu em $84,2 \%$ dos medicamentos, valor este semelhante ao observado pela OPAS $(84,2 \%)^{3}$, porém acima do estudo realizado por Santos \& Nitrini $(30,6 \%)^{13}$, Carneiro et al. $(33,9 \%)^{16}$, Marcondes $(71,0 \%)^{15}$ e Naves \& Silver $(73,2 \%)^{14}$.

Embora os resultados apresentem variações, para o setor público é obrigatória a prescrição de medicamentos pela Denominação Comum Brasileira - DCB, pois facilita o acesso aos medicamentos, seja através da compra ou de forma gratuita nos serviços de saúde. Um dos fatores que in- fluenciam a prescrição médica pelo nome de marca ou fantasia do medicamento é a indústria farmacêutica realizar um forte marketing para o incremento do consumo, além de disponibilizar várias apresentações medicamentosas para o mesmo princípio ativo ${ }^{4}$.

A OMS recomenda que $100 \%$ das prescrições sejam realizadas a partir da lista de medicamentos essenciais. Neste estudo, 91,9\% dos medicamentos prescritos seguiram a Relação Municipal de Medicamentos Essenciais (REMUME). Este dado é superior ao de Carneiro et al., que cita $54,1 \%^{16}$, Colombo et al, $82,4 \%^{7}$, Santos \& Nitrini, $83,4 \%^{13}$, Naves \& Silver, $85,3 \%^{14}$, Marcondes, $87,0 \%{ }^{15}$, e ao da OPAS $78,3 \%{ }^{3}$.

Pode-se atribuir o valor deste indicador è entrega da REMUME, em abril de 2005, a todas as unidades de saúde atendidas pela FACEN, assim como à requisição de medicamentos realizada em formu- 
lário padrão contendo os medicamentos padronizados.

Dos 2.166 medicamentos, $21,1 \%$ eram antibióticos, apresentando-se próximo ao considerado ideal pela OMS, $20,0 \%$ ou menos ${ }^{10}$. O valor encontrado assemelha-se ao de Santos \& Nitrini, $(21,3 \%)^{13}$, realizado em Ribeirão Preto, SP. Mas é superior ao obtido por Colombo et al., $12,5 \%^{7}$ e se apresenta inferior ao de Naves \& Silver, $26,4 \%^{14}$, Carneiro et al., 31,3\% ${ }^{16}$; Marcondes, $(33,0 \%)^{15}$ e pela OPAS, $40,1 \%{ }^{3}$.

O principal problema com relação ao uso indiscriminado e abusivo de antibióticos é o desenvolvimento de bactérias resistentes, que, em futuro próximo, necessitará da administração de antibióticos mais potentes e, provavelmente, mais onerosos no tratamento de infecções causadas pelos mesmos microorganismos.

Apenas 1,1\% dos medicamentos prescritos eram injetáveis. Este valor apresenta-se muito inferior ao obtido nos outros estudos, onde Colombo et al. verificaram 8,1\% ${ }^{7}$, Carneiro et al., 9,9\% ${ }^{16}$, Marcondes, $13,0 \%{ }^{15}$, Naves \& Silver, $7,5 \%^{14}$, Santos \& Nitrini, 8,3\% ${ }^{13}$. Na avaliação da assistência farmacêutica brasileira, o valor verificado foi $7,9 \%^{3}$.

Como o estudo não se propôs a buscar as causas do uso das diferentes formas farmacêuticas, não temos explicação para este valor bastante baixo de uso de injetáveis. No entanto, é plausível se supor que tal disparidade pode ser conseqüência do período em que o estudo foi desenvolvido, época quente do ano, quando a população é menos exposta a doenças. Outra possibilidade seria a procura direta da população de serviços de saúde especializados ou de urgência, quando é necessária a administração de injetáveis, o que impossibilitaria a coleta de dados.

Importante ressaltar que as principais políticas de medicamentos no país são recentes: em 1998 ocorreu a publicação da Política Nacional de Medicamentos (PNM), em 2003 foi realizada a Conferência Nacional de Medicamentos e Assistência Farmacêutica (CNMAF) e, em 2004, foi promulga- da a Política Nacional de Assistência Farmacêutica (PNAF). Dessa forma, alguns trabalhos realizados há alguns anos, como o de Santos \& Nitrini ${ }^{13}$ e Carneiro et al. ${ }^{16}$, não puderam refletir diretrizes hoje implantadas nos municípios brasileiros.

Seguindo a ATC, o grupo farmacológico mais prescrito foi antibióticos $(21,0 \%)$, em seguida os antiparasitários $(18,4 \%)$, analgésicos e antipiréticos (15,4\%), medicamentos para o aparelho digestivo $(9,5 \%) \mathrm{e}$ respiratório $(9,2 \%)$ (Figura 2$)$.

Como não há padrão de prescrição, pois esta ocorre de acordo com as patologias apresentadas pela população, os resultados encontrados diferem de outros estudos. Naves \& Silver (2005) verificaram que os medicamentos para o aparelho cardiovascular foram os mais prescritos $(26,8 \%)$, seguido dos antimicrobianos $(13,1 \%)$ e analgésicos $(8,9 \%)^{14}$. Colombo verificou que as classes terapêuticas mais prevalentes foram os analgésicos (14,3\%), os antibacterianos sistêmicos (12,5\%) e os antiinflamatórios $(12,5 \%)^{7}$.

Neste estudo faz-se interessante ressaltar que foram poucas as prescrições de medicamentos para o aparelho cardiovascular $(1,6 \%)$, embora as doenças desse sistema sejam uma das mais prevalentes na sociedade. Atribuiu-se este fato ao programa de Hipertensão e Diabetes (HiperDia), do Ministério da Saúde, que realiza o acompanhamento dos pacientes e distribui a medicação para cada usuário, sendo a mesma registrada no cartão do programa.

Uma limitação do estudo foi a impossibilidade da coleta de dados nas 20 unidades, conforme mencionado anteriormente, havendo assim perda no número de unidades pesquisadas. Outro procedimento que pode se constituir um viés para a pesquisa foi a solicitação da receita médica em duas vias, pois os profissionais prescritores podem ter tido maior cuidado no momento da prescrição e, assim, tornar os indicadores encontrados mais positivos. Contudo, entende-se que tais circunstâncias não tornam inválidos os resultados obtidos, possibilitando que a 
pesquisa apresente importantes informações para a avaliação da assistência à saúde prestada à população.

\section{Conclusões}

Tornou-se evidente que os medicamentos mais relevantes para as Unidades Básicas de Saúde da Família de Campina Grande são os antibióticos, antiparasitários, analgésicos e antipiréticos, e os medicamentos destinados a tratamentos do aparelho digestivo e respiratório. Portanto, faz-se importante para os serviços de saúde conhecer as principais demandas da comunidade, a fim de realizar as devidas intervenções e assim adequar suas atividades às necessidades da população.

Conforme apresentado, percebe-se que os indicadores de prescrição de antibióticos e medicamentos pelo nome genérico em Campina Grande apresentam - se similares aos de outros locais do país. No entanto, foram constatados melhores índices para a prescrição de injetáveis, medicamentos da REMUME e a média de medicamentos por receita. Tais índices podem ser considerados conseqüências do aumento das discussões sobre o assunto, em virtude da publicação e divulgação da Política Nacional de Medicamentos e da realização da Conferência Municipal de Medicamentos e Assistência Farmacêutica.

\section{Referências}

1. BRASIL, Ministério da Saúde. Gestão Municipal de Saúde: leis, normas e portarias atuais. Rio de Janeiro: Brasil: Ministério da Saúde; 2001.

2. BRASIL, Ministério da Saúde. Portaria GM no 3.916, de 30 de outubro de 1998. Aprova a Política Nacional de Medicamentos. Diário Oficial da União. Brasília, s.1, n.215-E, p.18, 10 de novembro de 1998.

3. OPAS, Organização Pan-Americana de Saúde. Avaliação da Assistência Farmacêutica no Brasil. Brasília: Organização Pan-Americana da Saúde: Ministério da Saúde; 2005.

4. Marin N, Luiza VL, Castro CGS, Santos SM. Assistência farmacêutica para gerentes municipais. Rio de Janeiro: OPAS/OMS; 2003.

5. OMS, Organização Mundial de saúde. How to investigate drug use in health facilities - selected drug use indicators. WHO. DAP 93.1; 1993.

6. Acurcio F. A, Perrini E, Magalhães SMS, Vieira Terceiro LG, Coutinho KEO, Caldeira Ket al. Analysis of medical prescriptions dispensed at health centers in Belo Horizonte, Minas Gerais, Brazil. Cad Saúde Pública 2004; 20(1): 72-9.

7. Colombo D, Helena ETS, Agostinho ACMG, Didjurgett JSMA. Padrão de Prescrição de Medicamentos nas Unidades de Programa Saúde da Família de Blumenau. Rev Bras Cien Farm 2004; 40 n. 4.

8. BRASIL, Conselho Nacional de Secretários de Saúde. Para entender a gestão do SUS. Brasília: Conass, 2003. 248 p.

9. Paim JS. Políticas de descentralização e atenção primária à Saúde. In: Rouquayrol MZ, Almeida Filho N. Epidemiologia \& Saúde. $5^{\text {a }}$ ed. Rio de Janeiro: MEDSI; 1999. p. 489-503.
10. PAHO, Pan American Health Organization. Rapid Pharmaceutical Management Assessment: an indicatorbased approach. Washignton: PAHO; 1995.

11. WHO, Collaborating Centre for Drugs Statistics Methodology Disponível em: http://www.whocc.no/ atcddd/ Acesso em 18 de junho de 2006.

12. BRASIL, Ministério da Saúde. Relação Nacional de Medicamentos Essenciais - RENAME. $3^{\text {a }}$ ed. Brasília: Ministério da Saúde; 2002.

13. Santos V, Nitrini SMOO. Indicadores do uso de medicamentos prescritos e de assistência ao paciente de serviços de saúde. Rev Saúde Pública 2004; 38(6): 819-26.

14. Naves JOS, Silver LD. Evaluation of pharmaceutical assistance public primary care in Brasília, Brazil. Rev Saúde Pública 2005; 39(2): 223-30.

15. Marcondes NSP. A assistência farmacêutica básica e o uso de medicamentos na zona urbana do município de Ponta Grossa, Paraná: um estudo de caso. Rio de Janeiro: FIOCRUZ/ENSP; 2002.

16. Carneiro RM, Marques MCP, Simões MJS. Estudo das prescrições de medicamentos em crianças de 0 a 2 anos atendidas no serviço municipal de saúde de Américo Brasiliense - SP, 1999. Rev Ciências Farmacêuticas. 2000; 21(2): 229-48.

Recebido em: 07/11/06 Versão final reapresentada em: 26/04/07 Aprovado em: 28/05/07 